\title{
PROJECTION OF ANALYTIC SETS AND BERNSTEIN INEQUALITIES
}

\author{
JEAN-PIERRE FRANÇOISE \\ Département de Mathématiques, Université de Paris VI, BP 172 \\ 4, Place Jussieu, tour 46-45, 5e étage, 75252 Paris, France \\ E-mail: jpf@ccr.jussieu.fr \\ Y. YOMDIN \\ Department of Theoretical Mathematics, The Weizmann Institute of Science \\ Rehovot 76100, Israel \\ E-mail:yomdin@wisdom.weizmann.ac.il
}

I. Bernstein inequality and the number of zeroes. We first give two definitions (cf. N. Roytvarf, Y. Yomdin $[\mathrm{R}-\mathrm{Y}]$ ). $\Delta_{R}$ denotes, as usual, the closed disk of radius $R$, centred at 0 .

Definition I.1. Let $R>0,0<\alpha<1$ and $K>0$ be given and let $f$ be holomorphic in a neighborhood of $\Delta_{R}$. We say that $f$ belongs to the Bernstein class $B_{R, \alpha, K}^{1}$ if

$$
\frac{\max \left\{|f(z)|, z \in \Delta_{R}\right\}}{\max \left\{|f(z)|, z \in \Delta_{\alpha R}\right\}} \leq K .
$$

Re mark. The name "Bernstein class" is justified by the fact that, according to one of the classical Bernstein inequalities, any polynomial of degree $d$ belongs to $B_{R, \alpha, K}^{1}$, $K=(1 / \alpha)^{d}$ for any $R$ and $\alpha$.

Definition I.2. Let a natural $N, R>0$ and $C>0$ be given, and let $f(z)=$ $\sum_{k=0}^{\infty} f_{k} z^{k}$ be an analytic function in a neighborhood of $0 \in \mathbb{C}$. We say that $f$ belongs to the Bernstein class $B_{N, R, C}^{2}$, if

$$
\left|f_{j}\right| R^{j} \leq C \max \left\{\left|f_{i}\right| R^{i}, i=0, \ldots, N\right\}, \quad j \geq N+1 .
$$

The two classes $B^{1}$ and $B^{2}$ essentially coincide. More precisely, we have the following

1991 Mathematics Subject Classification: 58F21, 13B21.

Received by the editors: November 5, 1996; in the revised form: July 13, 1998.

The paper is in final form and no version of it will be published elsewhere. 
Lemma I.3. Let $f$ be an element of $B_{N, R, C}^{2}$. Then $f$ is analytic in an open disk $\stackrel{\triangle}{R}_{R}$ and for any $R^{\prime}<R, 0<\alpha<1$ and $K=\left(\frac{1}{\alpha}\right)^{N}\left[1+\left(1-\alpha^{N}\right) \frac{\alpha}{1-\alpha}+C \frac{\beta}{1-\beta}\right], \beta=R^{\prime} / R$, $f$ belongs to $B_{R^{\prime}, \alpha, K}^{1}$.

Proof. The convergence of $f(z)=\sum_{k=0}^{\infty} f_{k} z^{k}$ on $\Delta_{R}$ is immediate. Let $m=$ $\max \left\{|f(z)|, z \in \Delta_{\alpha R^{\prime}}\right\}$. Then by the Cauchy formula, $\left|f_{i}\right| \leq m /\left(\alpha R^{\prime}\right)^{i}$ for any $i$. In particular, $\left|f_{i}\right| R^{i} \leq m /\left(\alpha R^{\prime} / R\right)^{i} \leq m /\left(\alpha R^{\prime} / R\right)^{N}$ for $i=0, \ldots, N$. Hence $\left|f_{j}\right| R^{j} \leq$ $C m /\left(\alpha R^{\prime} / R\right)^{N}$ for any $j \geq N+1$. Now we can estimate $|f|$ on $\Delta_{R^{\prime}}$ as follows:

$$
\begin{aligned}
\max \left\{|f(z)|, z \in \Delta_{R^{\prime}}\right\} & \leq \sum_{k=0}^{N}\left|f_{k}\right| R^{\prime k}+\sum_{k=N+1}^{\infty}\left|f_{k}\right| R^{\prime k} \\
\leq m \sum_{k=0}^{N}\left(\frac{1}{\alpha R^{\prime}}\right)^{k} R^{\prime k} & +\frac{C m}{\left(\alpha R^{\prime} / R\right)^{N}} \sum_{k=N+1}^{\infty}\left(R^{\prime} / R\right)^{k} \\
& =m\left(\frac{1}{\alpha}\right)^{N}\left[1+\left(1-\alpha^{N}\right) \frac{\alpha}{1-\alpha}+C \frac{\beta}{1-\beta}\right] .
\end{aligned}
$$

R e m a r k. The constant $K$ in Lemma I.3 can be chosen as $\left(\frac{1}{\alpha}\right)^{N}\left(1+C \frac{\alpha \beta}{1-\alpha \beta}+C \frac{\beta}{1-\beta}\right)$ which in some cases gives a better estimate.

Conversely, if $f$ belongs to $B_{R, \alpha, K}^{1}$, then it belongs to $B_{N, R, C}^{2}$ with $N=\log _{\alpha} K$ and $C$ given explicitly through $R, \alpha, K$ (cf. N. Roytvarf, Y. Yomdin [R-Y], Hayman [Ha]).

A relevance of Bernstein classes to our purpose is explained by the following lemma (which is well known in different forms in various fields of complex analysis; we give a version, obtained by $\mathrm{M}$. Waldschmidt [W] in relation to transcendent number theory).

LEMMA I.4. Let $R>0$, and $0<\alpha<1$ be given and let $f$ be holomorphic in a neighborhood of $\Delta_{R}$. Then the number of zeroes of $f$ in $\AA_{\alpha R}$ does not exceed

$$
\frac{\log \left(\max \left\{|f(z)|, z \in \Delta_{R}\right\} / \max \left\{|f(z)|, z \in \Delta_{\alpha R}\right\}\right)}{\log \left[\left(1+\alpha^{2}\right) / 2 \alpha\right]} .
$$

In other words, for an element $f$ of $B_{R, \alpha, K}^{1}$,

$$
\#\left\{f^{-1}(0) \cap \Delta_{\alpha R}\right\} \leq \frac{\log K}{\log \left[\left(1+\alpha^{2}\right) / 2 \alpha\right]} .
$$

Frequently, in the theory of differential equations, we deal with analytic developments $f(z)=\sum_{k=0}^{\infty} f_{k} z^{k}$ where $f_{k}$ is defined inductively by an expression which involves the preceding coefficients. So it can often be shown that $f$ belongs to a certain Bernstein class $B^{2}$. Combining the above results, we can estimate the number of zeroes of the functions in $B^{2}$ as follows:

Proposition I.5. Let $f$ be an element of $B_{N, R, C}^{2}$. Then for any $R^{\prime \prime}<R$, the number of zeroes of $f$ in ${\stackrel{\circ}{R^{\prime \prime}}}_{\text {does not exceed }}$

$$
N \cdot \min _{\left\{\alpha,\left(R^{\prime \prime} / R\right)<\alpha<1\right\}} \frac{1+\log \left(1+\left(1-\alpha^{N}\right) \frac{\alpha}{1-\alpha}+C \frac{\gamma}{1-\gamma}\right) / \log (1 / \alpha)}{1+\log \left(\left(1+\alpha^{2}\right) / 2\right) / \log (1 / \alpha)},
$$

where $\gamma=R^{\prime \prime} / \alpha R<1$. 
Proof. For any $\alpha, R^{\prime \prime} / R<\alpha<1$, let $R^{\prime}=R^{\prime \prime} / \alpha$. Then by Lemma I.3, $f$ belongs to $B_{R^{\prime}, \alpha, K}^{1}$, with $K=\left(\frac{1}{\alpha}\right)^{N}\left[1+\left(1-\alpha^{N}\right) \frac{\alpha}{1-\alpha}+C \frac{\gamma}{1-\gamma}\right]$, where $\gamma=R^{\prime \prime} / \alpha R=R^{\prime} / R$. Hence, by Lemma I.4 the number of zeroes of $f$ on $\AA_{R^{\prime \prime}}=\stackrel{\triangle}{\alpha}_{\alpha R^{\prime}}$ is bounded by

$$
\begin{aligned}
& \frac{\log (1 / \alpha)^{N}\left(1+\left(1-\alpha^{N}\right) \frac{\alpha}{1-\alpha}+C \frac{\gamma}{1-\gamma}\right)}{\log \left(\left(1+\alpha^{2}\right) / 2 \alpha\right)} \\
& \quad=N \cdot \frac{1+\log \left(1+\left(1-\alpha^{N}\right) \frac{\alpha}{1-\alpha}+C \frac{\gamma}{1-\gamma}\right) / \log (1 / \alpha)}{1+\log \left(\left(1+\alpha^{2}\right) / 2\right) / \log (1 / \alpha)} .
\end{aligned}
$$

Since the value of $\alpha$ between $\left(R^{\prime \prime} / R\right)$ and 1 or, equivalently, the value of $R^{\prime}, R>R^{\prime}>$ $R^{\prime \prime}$ can be chosen arbitrarily, the proposition follows.

Corollary I.6. Let $f$ be an element of $B_{N, R, C}^{2}$. Then

1) For $R^{\prime \prime}=R / 4$, the number of zeroes of $f$ on $\triangle_{R^{\prime \prime}}$ does not exceed $N \log _{5 / 4}(4+2 C)$.

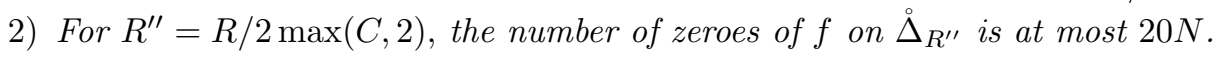

3) For $R^{\prime \prime}=R e^{-(10 N+2)} / \max (C, 2)$, this number is at most $N$.

Proof. To prove 1), take $\alpha=\frac{1}{2}$. Then $\gamma=\frac{1}{2}$ and

$$
\#\left\{f^{-1}(0) \cap \Delta_{R^{\prime \prime}}\right\} \leq N \cdot \frac{1+\log \left(1+\left(1-\left(\frac{1}{2}\right)^{N}\right)+C\right) / \log 2}{1+\log \frac{5}{8} / \log 2} \leq N \log _{5 / 4}(4+2 C) .
$$

In 2) we also choose $\alpha=\frac{1}{2}$. Then $\gamma=1 / \max (C, 2)$, and we get

$$
\#\left\{f^{-1}(0) \cap \Delta_{R^{\prime \prime}}\right\} \leq N \cdot \frac{1+\log \left(1+\left(1-\left(\frac{1}{2}\right)^{N}\right)+2\right) / \log 2}{1+\log \frac{5}{8} / \log 2} \leq 20 N .
$$

Finally, for $R^{\prime \prime}=R e^{-(10 N+2)} / \max (C, 2)$, we put $\alpha=e^{-10 N}$; then $\gamma=1 / e^{2} \max (C, 2)$, and $\#\left\{f^{-1}(0) \cap \Delta_{R^{\prime \prime}}\right\} \leq N \cdot(1+(2 / 3 N))$. Since the number of zeroes is an integer, this yields $\#\left\{f^{-1}(0) \cap \Delta_{R^{\prime \prime}}\right\} \leq N$.

Remark. By taking into account the Bernstein inequality, the last conclusion is strong enough to prove that the number of zeroes of a polynomial of degree $d$ does not exceed $d$.

II. Projection of analytic sets. We introduce now the algebra $\mathbb{C}(R)$ as follows:

$$
f(\boldsymbol{x}, z)=f_{\boldsymbol{x}}(z)=f\left(x_{1}, \ldots, x_{n} ; z\right)=\sum_{k=0}^{\infty} z^{k} f_{k}\left(x_{1}, \ldots, x_{n}\right)
$$

belongs to $\mathbb{C}(R)$ if there are $(\alpha, \beta)$ so that the coefficients $f_{k}\left(x_{1}, \ldots, x_{n}\right)(k=0,1, \ldots)$ are polynomials in $\boldsymbol{x}=\left(x_{1}, \ldots, x_{n}\right)$ of degree less than $\alpha k+b$ and if $\sum_{k=0}^{\infty} R^{k}\left|f_{k}\right|<\infty$. The norm $\left|f_{k}\right|$ of the polynomial $f_{k}$ is (for instance) the sum of the absolute value of its coefficients.

Definition II.1. We define the Bautin ideal $I$ of $f(\boldsymbol{x}, z)$ as the ideal generated by the coefficients $f_{k}\left(x_{1}, \ldots, x_{n}\right)$. The Bautin index is the minimal integer $N$ so that $f_{0}\left(x_{1}, \ldots, x_{n}\right), \ldots, f_{N}\left(x_{1}, \ldots, x_{n}\right)$ generate this ideal $I$. 
We fix a total ordering $<$ on $\mathbb{N}^{n}$ so that:

$$
\begin{aligned}
& \alpha \in \mathbb{N}^{n}, \beta \in \mathbb{N}^{n}, \beta \neq 0, \text { then } \alpha<\alpha+\beta \\
& \alpha \in \mathbb{N}^{n}, \beta \in \mathbb{N}^{n}, \gamma \in \mathbb{N}^{n}, \alpha<\gamma \text { then } \alpha+\beta<\gamma+\beta .
\end{aligned}
$$

There are several possible choices of such an ordering. For instance, we can choose $<$ as follows: Let $C(\alpha)=\sum_{i=1}^{n} \alpha_{i}, \alpha<\beta$ if $C(\alpha)<C(\beta)$ or if $C(\alpha)=C(\beta)$ and there exists $k, 1 \leq k \leq n$, such that $\alpha_{j}=b_{j}$ for $j<k$ and $\alpha_{k}<\beta_{k}$.

Given a polynomial $f=\sum_{A \in \mathbb{N}^{n}} f_{A} x^{A}$, and the total ordering on $\mathbb{N}^{n}$, we denote by $\exp f$ the largest exponent $A$ such that $f_{A} \neq 0$. Let $\exp I=\left\{A \in \mathbb{N}^{n}, A=\exp f, f \in I\right\}$. There is a unique minimal set $E=\left\{E_{1}, E_{2}, \ldots, E_{d}\right\}$ such that for every element $\beta$ of $\exp I$, there is an element $\varepsilon$ in $E$ and an element $\alpha$ in $\mathbb{N}^{n}$ such that $\beta=\varepsilon+\alpha$.

If the ordering of the multi-indices is given, we call a set of elements $\left\{h_{1}, \ldots, h_{d}\right\}$ of $I$ such that $\exp h_{k}=E_{k}$ a standard basis (or Gröbner basis) of the ideal $I$.

Let $\rho=\max \left\{\left|h_{i}-x^{E_{i}}\right|, i=1, \ldots, d\right\}$.

TheOrem II.2. There exists $C>0$, depending on $f$, such that the function $\sum_{k=0}^{\infty}\left|f_{k}\right| z^{k}$ belongs to $B_{N, R /(1+\rho)^{n \alpha}, C}^{2}$.

Proof. Let $h_{1}\left(x_{1}, \ldots, x_{n}\right), \ldots, h_{d}\left(x_{1}, \ldots, x_{n}\right)$ be a Gröbner basis of the ideal $I$. Moreover, since $f_{0}, \ldots, f_{N}$ generate $I$, we have $h_{j}=\sum_{i=0}^{N} \phi_{i}^{j} f_{i}$. Let $C_{1}=\max _{i, j}\left|\phi_{i}^{j}\right|$.

From classical estimates on division of polynomials by an ideal, we obtain that there is $C_{2}>0$ such that for any element $h$ of the ideal $I$,

$$
h=\sum_{j=1}^{d} g_{j} h_{j}, \text { with }\left|g_{j}\right| \leq C_{2}|h|(1+\rho)^{n \operatorname{deg} h} .
$$

Several generalizations of these crucial estimates have been produced in the setting of analytic coefficients (cf. [Br], [H], [Ga]). Hence,

$$
h=\sum_{i=0}^{N} g_{i}^{\prime} f_{i}, \text { with }\left|g_{i}^{\prime}\right| \leq d C_{1} C_{2}|h|(1+\rho)^{n \operatorname{deg} h} .
$$

In particular, for any $j \geq N+1$ we have

$$
f_{j}=\sum_{i=0}^{N} g_{i}^{\prime j} f_{i}, \text { with }\left|g_{i}^{\prime j}\right| \leq d C_{1} C_{2}\left|f_{j}\right|(1+\rho)^{n(\alpha j+\beta)} \leq d C_{1} C_{2} C_{3}\left((1+\rho)^{n \alpha} / R\right)^{j}
$$

since for an element $f(\boldsymbol{x}, z)=\sum_{k=0}^{\infty} z^{k} f_{k}\left(x_{1}, \ldots, x_{n}\right)$ of $\mathbb{C}(R)$, there is a constant $C_{3}$ such that $\left|f_{j}\right| \leq C_{3}$. Denote $d C_{1} C_{2} C_{3}(1+\rho)^{n \beta}$ by $C_{4}$. This yields

$$
\left|f_{j}\right| \leq \sum_{i=0}^{N}\left|g_{i}^{\prime j}\right|\left|f_{i}\right| \leq C_{4}\left((1+\rho)^{n \alpha} / R\right)^{j} \sum_{i=0}^{N}\left|f_{i}\right| \text {. }
$$

We obtain:

$$
\left|f_{j}\right| R^{\prime} j \leq C_{4}(N+1) \max \left\{\left|f_{i}\right|, i=0, \ldots, N\right\} \leq C_{5} \max \left\{\left|f_{i}\right| R^{\prime i}, i=0, \ldots, N\right\},
$$

where $C_{5}=C_{4} \max \left(1 / R^{\prime N}, 1\right), R^{\prime}=R /(1+\rho)^{n \alpha}$.

This proves Theorem II.2 with $C=C_{5}$. 
TheOREM II.3. Let $f$ be an element of $\mathbb{C}(R)$. Assume that there exist $\alpha, \beta$ so that the coefficients $f_{k}\left(x_{1}, \ldots, x_{n}\right)$ are homogeneous of degree $\alpha k+\beta$. Then for all $\boldsymbol{x}$, the series $f(\boldsymbol{x} ; z)=\sum_{k=0}^{\infty} z^{k} f_{k}(x)$ belongs to $B_{N, R^{\prime} /|\boldsymbol{x}|^{\alpha}, C|\boldsymbol{x}|^{\beta}}^{2}$.

Write

$$
f_{j}(\boldsymbol{x})=\sum_{i=0}^{N} g_{i}^{\prime j}(\boldsymbol{x}) f_{i}(\boldsymbol{x})
$$

Denote by $\operatorname{hom}_{i}(p(x))$ the homogeneous component of degree $i$ of a polynomial $p(x)$. We obtain

$$
f_{j}(\boldsymbol{x})=\sum_{i=0}^{N} \operatorname{hom}_{\alpha(j-i)+\beta}\left(g_{i}^{\prime j}(\boldsymbol{x})\right) f_{i}(\boldsymbol{x}) .
$$

Then we get

$$
\left|\operatorname{hom}_{\alpha(j-i)+\beta}\left(g_{i}^{\prime j}(x)\right)\right| \leq\left|g_{i}^{\prime j}\right||\boldsymbol{x}|^{\alpha(j-i)+b} \leq d C_{1} C_{2} C_{3}\left(1 / R^{\prime}\right)^{j}|\boldsymbol{x}|^{\alpha(j-i)+\beta},
$$

and this yields

$$
\left|f_{j}(\boldsymbol{x})\right| \leq \sum_{i=0}^{N} d C_{1} C_{2} C_{3}\left(1 / R^{\prime}\right)^{j}|\boldsymbol{x}|^{\alpha(j-i)+\beta}\left|f_{i}(\boldsymbol{x})\right| \leq C_{4}|\boldsymbol{x}|^{\beta}\left(|\boldsymbol{x}|^{\alpha} / R^{\prime}\right)^{j} \sum_{i=0}^{N}\left|f_{i}(\boldsymbol{x})\right| /|\boldsymbol{x}|^{\alpha i} .
$$

Hence,

$$
\left|f_{j}(\boldsymbol{x})\right|\left(R^{\prime} /|\boldsymbol{x}|^{\alpha}\right)^{j} \leq C|\boldsymbol{x}|^{\beta} \max \left\{\left|f_{i}(\boldsymbol{x})\right|\left(R^{\prime} /|\boldsymbol{x}|^{\alpha}\right)^{i}, i=0, \ldots, N\right\} .
$$

Theorem II.4. Let $f$ be an element of $\mathbb{C}(R), N, C$ be as above. Let $R^{\prime}(\boldsymbol{x})=\frac{1}{4} R^{\prime} /|\boldsymbol{x}|^{\alpha}$, $R^{\prime \prime}(\boldsymbol{x})=\left(R /|x|^{\alpha}\right) / 2 \max \left(C|\boldsymbol{x}|^{\beta}, 2\right), R^{*}(\boldsymbol{x})=\left(R /|\boldsymbol{x}|^{\alpha}\right) e^{-(10 N+2)} / \max \left(C|\boldsymbol{x}|^{\beta}, 2\right)$. Then for any $\boldsymbol{x}$, the function $f_{\boldsymbol{x}}(z)$ can have on the disks $\Delta_{R^{\prime}(x)},{\stackrel{\Delta}{R^{\prime \prime}(x)}},{\stackrel{\Delta}{R^{*}(x)}}$, at most $N \log _{5 / 4}\left(4+2 C|\boldsymbol{x}|^{\beta}\right), 20 N$ and $N$ zeroes, respectively.

In the article $[\mathrm{F}-\mathrm{Y}]$, we followed a different presentation based on the use of the norm "maximum on a polydisc". This allows to handle more general data ( $f$ may have analytic coefficients) but it is necessary to use priviliged neighborhoods.

The Łojasiewicz inequality appears closely related to the subject. We take the opportunity of this Symposium to mention briefly the connection and postpone its developments to further studies.

The Łojasiewicz inequality entails a constant $K$ and an exponent $\delta$ so that

$$
K\left(\sum_{k=0}^{\infty} f_{k}^{2}(\boldsymbol{x})\right)^{\delta} \leq \sum_{i=0}^{N} f_{i}^{2}(\boldsymbol{x}) .
$$

It yields the inequality

$$
\left|f_{j}(\boldsymbol{x})\right| \leq C \max \left\{\left|f_{i}(\boldsymbol{x})\right|^{1 / \delta}, i=0, \ldots, N\right\}, \quad j \geq N+1 .
$$

Going back to the Jensen inequality, we obtain the same type of bound for the number of zeroes (cf. Lemma I.4). The only change is that this bound gets multiplied by $1 / \delta$.

The authors thank warmly E. Bierstone and P. Milman for several discussions which improved the presentation of their results. We like to add the reference [E-T-Z], which recently appeared, where the most general case (codimension $\geq 1$ ) has been considered with different techniques. 


\section{References}

[B] N. N. Bautin, On the number of limit cycles which appear with the variation of the coefficients from an equilibrium position of focus or center type, Mat. Sbornik 30 (1952), 181-196 (in Russian).

[Br] J. Briançon, Weierstrass préparé à la Hironaka, Astérisque, 7-8 (1973), 67-73.

[Bu] B. Buchberger, Ein algorithmisches Kriterium für die Lösbarkeit eines algebraischen Gleichungssystems, Aequationes Math. 4 (1970), 374-383.

[F-Y] J.-P. Françoise, Y. Yomdin, Bernstein inequality and applications to differential equations and analytic geometry, J. Funct. Anal. 146 (1997), 185-205.

[Ga] A. Galligo, Sur le théorème de préparation de Weierstrass, Astérisque 7-8 (1973), 165-169.

[Ha] W. K. Hayman, Differential inequalities and local valency, Pacific J. Math. 44 (1973), 117-137.

[H] H. Hironaka, Characteristic polyhedra of singularities, J. Math. Kyoto Univ. 7 (1967), 251-293.

[€] S. Łojasiewicz, Ensembles semi-analytiques, Inst. de Hautes Études Scientifiques, Bures-sur-Yvette, 1965.

[Ł-T-Z] S. Łojasiewicz, J. C. Tougeron, M. A. Zurro, Éclatement des coefficients des séries entières et deux théorèmes de Gabrielov, Manuscripta Math. 92 (1997), 325-337.

[M] G. Maltsiniotis, Privilège numérique uniforme, Astérisque 194-195 (1991).

[R-Y] N. Roytvarf, Y. Yomdin, Bernstein classes, to appear.

[W] M. Waldschmidt, Nombres transcendants, Lecture Notes in Math. 402, Springer, Berlin, 1974. 